\author{
П.А. Брыгин*, С.В. Журавель, Д.А. Троицкий, И.И. Уткина \\ ГБУЗ «НИИ скорой помощи им. Н.В. Склифосовского ДЗМ», \\ 129090, Россия, Москва, Большая Сухаревская площадь, д. 3
}

*Контактная информация: Павел Александрович Брыгин, канд. мед. наук, заведующий отделением реанимации для ожоговых больных НИИ скорой помощи им. Н.В. Склифосовского, e-mail: p.brygin@gmail.com

В статъе обсуждается проблема прогнозирования возможности восстановления ббункиии легких при остром респираторном дистресс-синдроме при проведении экстракорпоральной мембранной оксигенации. Рассмотрены данные международных исследований CESAR и EOLIA, посвященных анализу исходов экстракорпоралъной мембранной оксигенаиии при остром респираторном дистресс-синдроме. Обсуждается противоречивость полученных результатов. Приведенъ прогностические шкалъ PRESERVE U PESP, разработанные на основании результатов этих исследований, обсуждается ограниченность применимости их при различных вариантах острого респираторного дистресс-синдрома. Мы предлагаем разделить предикторы исхода применения экстракорпоральной мембранной оксигенации при остром респираторном дистресс-синдроме на 4 группь: 1. Критерии тяжести поражения легких, включаюшие параметры кислородотранспортной функиии легких и показатели механических свойств легких. 2. Время развития острой дыхательной недостаточности и начала экстракорпоральной мембранной оксигенации, отражающие как скорость развития патологических процессов в легких, так и оптилальность принятия решения о начале экстракорпоральной мембранной оксигенаиии. 3. Этиология легочных нарушений, напрямую влияюшая на обратилость патологических процессов в легких. 4. Тяжесть общего состояния больного, включающая выраженность проявлений полиорганной недостаточности, степень декомпенсации сопутствуюших хронических заболеваний, в том иисле - онокологических и связанных с имлуносупрессией. Определеннъе заболевания сопровождаются повышенным риском специбических осложнений, связанных с проведением процедуры әкстракорпоральной мембранной оксигенации, в частности - геморрагических.

Ключевые слова: экстракорпоральная мембранная оксигенация, острый респираторный дистресс-синдром, острая дыхательная недостаточность

КОНФликт интеРЕсов Авторы заявляют об отсутствии конфликта интересов

ФинансиРованиЕ Исследование проводилось без спонсорской поддержки

Благодарности Авторы выражают благодарность Сергею Сергеевичу Петрикову, чл.-корр. РАН, д-ру мед. наук, директору ГБУЗ «НИИ скорой помощи им. Н.В. Склифосовского ДЗМ», за научную консультацию и исправления в процессе написания рукописи

Брыгин П.А., Журавель С.В., Троицкий Д.А., Уткина И.И. Предикторы эффективности применения экстракорпоральной мембранной оксигенации при острой дыхательной недостаточности. Трансплантология. 2020;12(3):220-230. https://doi.org/10.23873/20740506-2020-12-3-220-230 


\title{
Predictors of extracorporeal membrane oxygenation efficacy in patients with acute respiratory failure
}

\author{
P.A. Brygin*, S.V. Zhuravel, D.A. Troitskiy, I.I. Utkina \\ N.V. Sklifosovsky Research Institute for Emergency Medicine, \\ 3 Bolshaya Sukharevskaya Sq., Moscow 129090 Russia \\ * Correspondence to: Pavel A. Brygin, Cand. Med. Sci., Head of the Intensive and Critical Care Unit for Burn Patients, \\ N.V. Sklifosovsky Research Institute for Emergency Medicine, e-mail: p.brygin @ gmail.com
}

The purpose of this article is to describe the problem of predicting the lung function recovery in patients with extracorporeal membrane oxygenation for acute respiratory distress syndrome. Data from CESAR and EOLIA clinical trials on the efficacy of extracorporeal membrane oxygenation in patients with acute respiratory distress syndrome have been reviewed and some controversial results discussed. The prognostic PRESERVE and RESP scores developed as prognostic tools on the basis of the results of these studies, are presented, the limitations of their applicability in various forms of acute respiratory distress syndrome are discussed. We propose to subdivide the predictors of the extracorporeal membrane oxygenation outcome in patients with acute respiratory distress syndrome into 4 following groups: 1 . Lung injury severity criteria, including parameters of their lung mechanical and functional properties. 2. Time from acute respiratory failure onset to extracorporeal membrane oxygenation initiation, which reflects the rate of pathological processes in lungs and timing of decision to initiate extracorporeal membrane oxygenation. 3. The etiology of pulmonary disorders, directly affecting the reversibility of pathological processes in the lungs. 4 . The severity of the patient's general condition, including the severity of manifestations of multiple organ failure, the degree of decompensation of concomitant chronic diseases, including oncological and associated with immunosuppression. Several diseases are associated with a higher risk of specific complications, particularly hemorrhagic, during extracorporeal membrane oxygenation.

Keywords: extracorporeal membrane oxygenation, acute respiratory distress syndrome, acute respiratory failure

CONFLICT OF INTERESTS Authors declare no conflict of interest

Financing The study was performed without external funding

Acknowledgments The authors express their gratitude to Sergey S. Petrikov, Dr. Med. Sci., Corr. Member of RAS, the Director of the N.V. Sklifosovsky Research Institute for Emergency Medicine, for his scientific advice and corrections proposed at stage of writing the manuscript

Brygin PA, Zhuravel SV, Troitskiy DA, Utkina II. Predictors of extracorporeal membrane oxygenation efficacy in patients with acute respiratory failure. Transplantologiya. The Russian Journal of Transplantation. 2020;12(3):220-230. (In Russ.). https://doi. org/10.23873/2074-0506-2020-12-3-220-230

дО - дыхательный объем

ИВЛ - искусственная вентиляция легких

КТ - компьютерная томография

ОДН - острая дыхательная недостаточность

ОРДС - острый респираторный дистресс-синдром

ОРИТ - отделение реанимации и интенсивной терапии

ЭКМО- экстракорпоральная мембранная оксигенация

$\mathrm{FiO}_{2}$ - фрракция кислорода во вдыхаемой смеси

\section{Введение}

Экстракорпоральная мембранная оксигенация (ЭКМО) - аппаратный метод поддержания жизни, позволяющий частично замещать функцию легких в случае их крайне тяжелого поражения. Выполняя протезирующую роль, ЭКМО не оказывает прямого лечебного воздействия на легочную ткань, и, соответственно, результат лечения полностью зависит от обратимости основного процесса в легких. Статья посвящена анализу литературных источников, описывающих предикторы выживания при проведении вено-венозного ЭКМО у пациентов с паренхиматозной легочной недостаточностью.

\author{
LIS - оценка повреждения легких \\ OI - индекс оксигенации \\ OSI - индекс оксигенации с использованием сатурации \\ $\mathrm{PaO}_{2}$ - парциальное напряжение кислорода в артериаль- \\ ной крови \\ PEЕР - положительное давление в конце выдоха \\ Pplat - давление плато \\ $\triangle \mathrm{P}$ (driving pressure) - разница давления между Pplat и PEEP
}

История применения

экстракорпоральной мембранной оксигенации при острой дыхательной недостаточности

В основе метода ЭКМО лежит применение специальной полимерной мембраны, позволяющей насыщать кислородом венозную кровь, протекающую через экстракорпоральный контур [1]. Принципы ЭКМО разработаны в 60-х годах $\mathrm{XX}$ века. Долгое время эта методика применялась в основном в педиатрической практике. Первое применение ЭКМО у взрослых датируется 1972 г. [2], а первое научное исследование на эту тему проведено в 1979 г. [3]. Результаты применения ЭКМО того времени поначалу разочаровывали врачей и ученых. 
Вновь интерес к технологии ЭКМО возродился относительно недавно - после успешного ее применения во время пандемии гриппа H1N1, развившейся в 2009 г., когда конвенциональная терапия - искусственная вентиляция легких оказалась малоэффрективной [4, 5]. Успех ЭКМО у этой категории больных подтолкнул клиницистов к исследованию эффективности методики при других заболеваниях, приводящих к возникновению острой легочной недостаточности. В настоящее время для ЭКМО найдено применение в терапии заболеваний как первичной пульмональной этиологии, так и экстрапульмональных, осложнившихся тяжелой респираторной недостаточностью [6]. Кроме того, описано использование ЭКМО в качестве «моста» к трансплантации легких и для сердечно-легочной реанимации $[7,8]$. Одним из наиболее частых показаний к проведению ЭКМО является острый респираторный дистресс-синдром (ОРДС). Эффективность применения метода при ОРДС оценена в исследованиях CESAR [9] и EOLIA [7]. Исследование CESAR показало достоверное уменьшение 90-дневной летальности в группе с ЭКМО. Исследование EOLIA не продемонстрировало статистически значимого межгруппового различия, несмотря на то, что летальность в группе с ЭКМО была на $13 \%$ ниже, чем в группе сравнения. Стоит отметить, что оба исследования имели существенные ограничения, возможно, оказавшие влияние на результаты. Например, в исследовании CESAR часть пациентов из группы ЭКМО получили конвенциональную терапию, а в группе сравнения отсутствовала стандартизация параметров искусственной вентиляции легких (ИВЛ). Исследование EOLIA было прекращено по достижению $75 \%$ от максимальной выборки, $28 \%$ пациентов с рефрактерной гипоксемией из группы сравнения были перемещены в группу ЭКМО. Кроме того, авторы предположили, что мощность исследования была недостаточной для достижения изначальной цели продемонстрировать $20 \%$ разницу в летальности между группами ЭКМО и сравнения. В то же время данные метаанализа, проведенного на основании ретроспективного клинического исследования CESAR и EOLIA, а также трех обсервационных исследований, свидетельствовали о снижении летальности при использовании ЭКМО у пациентов с тяжелым ОРДС [10]. В целом противоречивость полученных данных не позволяла сделать однозначного вывода относительно пользы ЭКМО для пациентов.
Прогностические шкалы эффективности экстракорпоральной мембранной оксигенации

ЭКМО является сложной и дорогостоящей методикой, при этом может сопровождаться рядом серьезных осложнений [11]. Поэтому перед ее проведением необходимо оценить критерии обратимости повреждения легких и прогноз заболевания в целом.

Для решения этой задачи предложены прогностические шкалы PRESERVE (2013 г.) (табл. 1) [12] и RESP (2014 г.) (табл. 2) [13]. Шкала PRESERVE базируется на анализе 140 пациентов с ОРДС, которым проводилась ЭКМО в период с 2008 по 2012 г. Оценивались параметры ИВЛ (давление плато (Pplat), фрракция кислорода во вдыхаемой смеси $\left(\mathrm{FiO}_{2}\right)$, положительное давление в конце выдоха (PEEP|), дыхательный объем (ДО), driving pressure - $\Delta \mathrm{P}$ ), фрункции легких (комплайенс, отношение $\mathrm{PaO}_{2} / \mathrm{FiO}_{2}$, сатурация артериальной крови), а также параметры кислотно-основного состояния крови, проведенные лечебные мероприятия, временные интервалы (поступление - перевод в отделение реанимации и интенсивной терапии (ОРИТ), поступление - начало ЭКМО, перевод в ОРИТ - начало ЭКМО, начало ИВЛ - начало ЭКМО). На основании корреляции вышеуказанных фракторов с летальностью была предложена шкала, содержащая 7 критериев: возраст (менее 45, 45-55, более 55 лет), индекс массы тела более 30, иммуноскомпрометированность, количество баллов по шкале SOFA более 12 , длительность ИВЛ более 6 дней, использование прон-позиции до ЭКМО, РЕЕР менее $10 \mathrm{~cm}$ вод.ст. и Pplat более 30 см вод.ст. Однако некоторые параметры, например, комплайенс, для которых была продемонстрирована корреляция с выживаемостью, оказались не включены в окончательные критерии шкалы.

Шкала RESP (табл. 2, рисунок) базируется на анализе 2355 пациентов из регистра ELSO, которым с 2000 по 2012 г. проводилась ЭКМО по поводу ОРДС. Регрессионный анализ этиологии ОРДС, режимов ИВЛ и $\mathrm{pCO}_{2}$ артериальной крови перед началом ЭКМО, а также ассоциированных патологических состояний, параметров ЭКМО, проведенных лечебных мероприятий и возраста пациентов в группах выживших и скончавшихся пациентов определил критерии, вошедшие в шкалу RESP.

Из всех существующих шкал RESP разработана на основе анализа самой крупной выборки пациентов. Однако, по мнению авторов, это может быть методологическим ограничением, так как 


\section{ОБ30РНЫЕ 由ТАТЬИ И ЛЕНЦИИ}

\section{REVIEW ABTIGLES AND LEGTURES}

за 12 лет появилось новое поколение аппаратов ЭКМО и изменилась тактика лечения пациентов с ОРДС, что могло повлиять на однородность выборки. С другой стороны, две трети пациентов были включены в исследование после 2009 г., что,

Таблица 1. Параметры, используемые в шкале PRESERVE, и соответствующие им баллы

Table 1. Parameters used in the PRESERVE score and their corresponding scoring points

\begin{tabular}{|l|c|}
\hline \multicolumn{1}{|c|}{ Параметр } & Число баллов \\
\hline Возраст (годы) & 0 \\
\hline \multicolumn{1}{|c|}{ менее 45} & 2 \\
\hline $45-55$ & 3 \\
\hline \multicolumn{1}{|c|}{ более 55 } & -2 \\
\hline Индекс массы тела более 30 & 2 \\
\hline Иммуноскомпрометированность & 1 \\
\hline Более 12 баллов по шкале SOFA & 1 \\
\hline Продолжительность ИВЛ более 6 сут & 1 \\
\hline $\begin{array}{l}\text { Прон-позиция не использовалась до на- } \\
\text { чала ЭКМО }\end{array}$ & 2 \\
\hline РЕЕР менее 10 см вод.ст. & 2 \\
\hline Давление плато более 30 см вод.ст. & $\mathbf{0 - 1 4}$ \\
\hline Общее количество баллов & \\
\hline
\end{tabular}

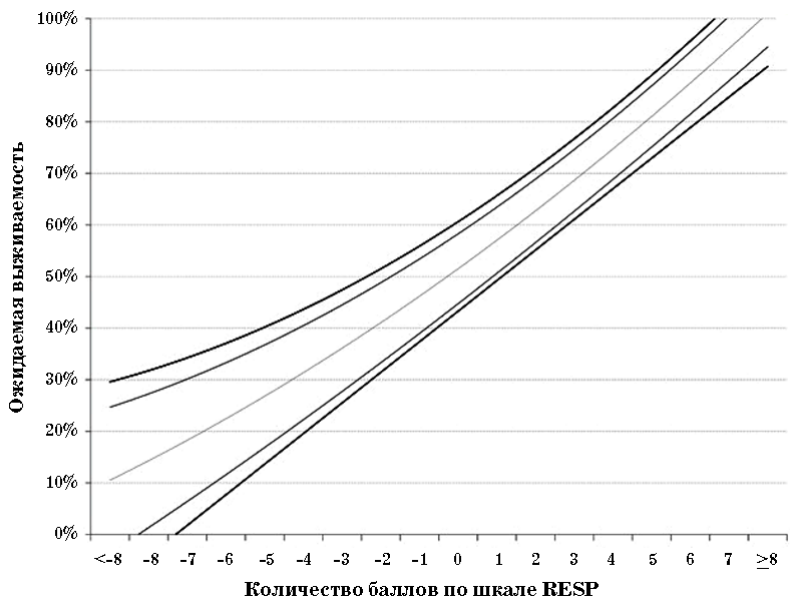

Рисунок. Ожидаемая выживаемость в зависимости от количества баллов по шкале RESP, светло-серые линии - границы 95\%-го доверительного интервала; темно-серые линии - границы 99\%-го доверительного интервала

Figure. Expected survival depending on the total RESP score; light-gray lines denote the limits of the $95 \%$ confidence interval; dark gray lines denote the limits of $99 \%$ confidence interval
Таблица 2. Параметры, используемые в шкале RESP, и соответствующие им баллы

Table 2. Parameters used in the RESP score and their corresponding scoring points

\begin{tabular}{|c|c|}
\hline Параметр & Число баллов \\
\hline \multicolumn{2}{|l|}{ Возраст (годы) } \\
\hline $18-49$ & 0 \\
\hline $50-59$ & -2 \\
\hline 60 и больше & -3 \\
\hline Иммуноскомпрометированный статус & -2 \\
\hline \multicolumn{2}{|l|}{ Продолжительность ИВЛ до начала ЭКМО } \\
\hline менее 48 ч & 3 \\
\hline от 48 ч до 7 сут & 1 \\
\hline более 7 сут & 0 \\
\hline \multicolumn{2}{|c|}{$\begin{array}{l}\text { Группа острого респираторного заболевания (выбрать } \\
\text { одну) }\end{array}$} \\
\hline Вирусная пневмония & 3 \\
\hline Бактериальная пневмония & 3 \\
\hline Бронхиальная астма & 11 \\
\hline Травма и ожоги & 3 \\
\hline Аспирационный пневмонит & 5 \\
\hline $\begin{array}{l}\text { Другое острое респираторное забо- } \\
\text { левание }\end{array}$ & 1 \\
\hline $\begin{array}{l}\text { Нереспираторное или хроническое } \\
\text { респираторное заболевание }\end{array}$ & 0 \\
\hline $\begin{array}{l}\text { Дисфункция центральной нервной си- } \\
\text { стемы }\end{array}$ & -7 \\
\hline $\begin{array}{l}\text { Острая ассоциированная (нереспиратор- } \\
\text { ная) инфрекция }\end{array}$ & -3 \\
\hline $\begin{array}{l}\text { Использование миорелаксантов до нача- } \\
\text { ла ЭКМО }\end{array}$ & 1 \\
\hline $\begin{array}{l}\text { Использование оксида азота до начала } \\
\text { ЭКМО }\end{array}$ & -1 \\
\hline Инфузия бикарбоната до начала ЭКМО & -2 \\
\hline $\begin{array}{l}\text { Остановка кровообращения до начала } \\
\text { ЭКМО }\end{array}$ & -2 \\
\hline \multicolumn{2}{|l|}{ Значение $\mathrm{PaCO}_{2}$, мм рт.ст. } \\
\hline менее 75 & 0 \\
\hline 75 и больше & -1 \\
\hline \multicolumn{2}{|l|}{ Значение пикового давления вдоха, см вод.ст. } \\
\hline менее 42 & 0 \\
\hline 42 и больше & -1 \\
\hline Общее число баллов & $-22-15$ \\
\hline
\end{tabular}

возможно, уменьшает ограничения, связанные с длительностью исследования. 
В руководствах по ЭКMO, например, ELSO Guidelines for Adult Respiratory Failure [14], ECMO In The Adult Patient [15], Extracorporeal Life Support For Adults [16] говорится о значимости оценки прогноза заболевания. В качестве очевидных предикторов неблагоприятного исхода указываются некурабельные состояния, такие, как необратимые повреждения головного мозга и терминальная стадия онкологического процесса.

\section{Прогностические критерии выживаемости при экстракорпоральной мембранной оксигенации \\ Прогностические критерии выживаемости можно разделить на 4 группы: \\ 1. Тяжесть поражения легких. \\ 2. Факторы времени развития острой дыха- тельной недостаточности и начала ЭКМО. \\ 3. Этиология легочных нарушений. \\ 4. Тяжесть общего состояния больного.}

Тяжесть поражения легких

К критериям тяжести поражения легочной паренхимы можно отнести показатели кислородтранспортной функции легких и параметры респираторной механики, отражающие податливость легочной ткани.

Тяжесть нарушений кислородтранспортной фрункции легких является основным показанием для начала процедуры ЭКМО. Руководства по ЭКМО [14-17], протоколы исследований [7, 9] и рекомендации по лечению ОР ДС [18] содержат в разделе «показания к ЭКМО» отношение $\mathrm{PaO}_{2} /$ $\mathrm{FiO}_{2}$, отражающее тяжесть гипоксемии и являющееся наиболее распространенным и простым в использовании критерием оценки тяжести легочного повреждения. Для получения более достоверных данных о тяжести дыхательной недостаточности рекомендуется оценивать отношение $\mathrm{PaO}_{2} / \mathrm{FiO}_{2}$ в динамике - при поступлении и через 24 ч [19]. Отношение $\mathrm{PaO}_{2} / \mathrm{FiO}_{2}$ также присутствует в качестве одного из критериев в шкале APPS, предложенной J. Villar et al. для прогнозирования исхода заболевания у пациентов с ОРДС [20]. С этой же целью предложено использовать модификацию отношения $\mathrm{PaO}_{2} / \mathrm{FiO}_{2}-\mathrm{OI}$ (индекс оксигенации), включающий кроме показателей $\mathrm{PaO}_{2}$ и $\mathrm{FiO}_{2}$ среднее давление в дыхательных путях. OI разработан в 1988 г. для определения показаний к ЭКМО у новорожденных с РДС [21], но оказался применимым для прогнозирования летальности при ОДН и во взрослой популяции [22]. Еще один показатель - индекс оксигенации с использованием сатурации (OSI), является моди- фрикацией индекса $\mathrm{SpO}_{2} / \mathrm{FiO}_{2}$ и также учитывает среднее давление в дыхательных путях. Доказана корреляция OSI с летальностью у пациентов с ОР ДС [23].

Шкала для оценки повреждения легких (LIS), предложенная J.F. Murray et al. [24] в 1988 г., также исследовалась в качестве прогностической модели. Однако полученные результаты не позволяют использовать LIS для прогнозирования исходов ввиду ее низкой чувствительности [25]. Сравнение индексов $\mathrm{PaO}_{2} / \mathrm{FiO}_{2}, \mathrm{SpO}_{2} / \mathrm{FiO}_{2}, \mathrm{OI}$, OSI и шкалы Murray LIS было проведено в ходе исследования VALID, которое продемонстрировало корреляцию между OI и OSI - оба показателя были выше у скончавшихся пациентов, но достоверная связь была продемонстрирована только для OSI. При этом $\mathrm{PaO}_{2} / \mathrm{FiO}_{2}, \mathrm{SpO}_{2} / \mathrm{FiO}_{2}$, OI и Murray LIS не являлись независимыми предикторами летальности [26].

Однако следует подчеркнуть, что тяжесть паренхиматозных легочных нарушений хоть и является неблагоприятным прогностическим критерием, не свидетельствует о необратимости повреждения и никогда не рассматривается как противопоказание к началу ЭКМО.

При разработке шкалы PRESERVE вообще показано отсутствие связи между выживаемостью и $\mathrm{PaO}_{2} / \mathrm{FiO}_{2}$ на момент начала ЭКМО.

Механические свойства легочной ткани, в первую очередь статическая податливость, являются важнейшей характеристикой состояния легких и вероятно могут служить прогностическим критерием обратимости патологического процесса. Лишь два исследования оценивали комплаенс респираторной системы как самостоятельный параметр: в исследовании Preserve значения легочного комплаенса в группе выживших были достоверно выше - медиана 19 в группе выживших и 16 в группе умерших, в исследовании M. Schmidt (2019) [27] также показана корреляция более высокой податливости легких с выживанием.

Другие работы не упоминают податливость легких в качестве прогностического критерия, но отмечают, что применение «жестких» режимов ИВЛ является неблагоприятным признаком. В исследовании L. Munshi et al. ИВЛ с пиковым давлением выше 30 см вод.ст. рассматривается как дополнительный фрактор повреждения легочной ткани и при длительности более 7 дней считается противопоказанием для начала ЭКМО [10].

В работах, посвященных ИВЛ при ОРДС, приводится множество доказательств тому, что 
высокое давление вдоха, $\Delta \mathrm{P}$, коррелируют с худшим исходом заболевания [28, 29]. Границами безопасной ИВЛ считается ДО в 6 мл/кг идеальной массы тела, $\Delta \mathrm{P}$ не более 15 см вод.ст. и пиковое давление вдоха не более 30 см вод.ст. [30].

Однако использование «непротективных» режимов ИВЛ не всегда является ошибкой врача. Применение высокого давления на вдохе, большого $\Delta \mathrm{P}$ может быть продиктовано нарушениями механических свойств легких, в частности - снижением податливости легочной ткани (compliance). В этом контексте остаются неясными причинно-следственные связи - использование «непротективных» режимов ИВЛ приводит к повреждению легких, или первичное нарушение податливости легочной ткани вынуждает использовать высокое давление вдоха и $\Delta \mathrm{P}$. Один и тот же ДО, считающийся «протективным» (6 мл/ кг идеальной массы тела), может быть получен при разном $\Delta \mathrm{P}$, в зависимости от податливости респираторной системы пациента. Если комплаенс снижен умеренно, будет обеспечено безопасное $\Delta \mathrm{P}$ на уровне $15 \mathrm{~cm}$ вод.ст. или ниже, однако при выраженных рестиктивных нарушениях тот же формально протективный дО будет реализован только со значительным превышением как $\Delta \mathrm{P}$, так и максимального давления вдоха при Pressure control или давления плато VC.

По данным M.B. Amato et al., уменьшение ДО и увеличение РЕEР приводили к лучшим исходам только если были ассоциированы с уменьшением $\Delta \mathrm{P}[31]$. То есть оценка ДО в контексте протективной вентиляции позволяет достоверно судить о связи ДО с выживаемостью и безопасностью ИВЛ только при учете комплаенса.

Как было продемонстрировано в исследовании H.S. Kim et al., включавшем 56 пациентов, которым проводилось ЭКМО по поводу паренхиматозной легочной недостаточности, в группе пациентов с неблагоприятным исходом показатель $\Delta \mathrm{P}$ до проведения ЭКМО был значительно выше, чем у выживших пациентов [32]. Вместе с тем были оценены показатели легочного комплаенса в обеих группах как до, так и во время ЭКМО, при этом у выживших пациентов комплаенс был выше как до проведения ЭКМО, так и во время ИВЛ при ЭКМО. Подобные результаты были получены M. Schmidt et al. в ходе исследования совместно с группой LIFEGARDS, где более низкий легочный комплаенс при инициации ЭКМО был связан с худшим исходом [27]. Однако то же исследование не показало достоверно значимых различий между показателями комплаенса в первые 2 дня на ЭКМО у выживших и умерших пациентов. Возможно, это связано с уменьшением комплаенса после начала протективной вентиляции легких при ЭКМО. Эта теория находит подтверждение в исследовании H. Roze et al., которое в 2016 г. продемонстрировало снижение торакопульмонального комплаенса в первые 24 ч после начала протективной ИВЛ [33]. Полученные результаты авторы связали с возможным дерекрутментом легких вследствие уменьшения ДО и предложили с целью предотвращения дерекрутмента при искусственном уменьшении ДО увеличивать РЕЕР.

Сходный вывод можно сделать на основании работы A.J. Walkey, результаты которой свидетельствовали о том, что у пациентов с ОРДС сочетание высоких значений РЕEР и низкого ДО ассоциировано с лучшим исходом, нежели у пациентов и низкими ДО и РЕEР [34].

Резюмируя все выше описанное, можно предположить, что именно величина податливости респираторной системы, а не раздельная оценка давления плато (Pinsp), $\Delta \mathrm{P}$ и ДО может отражать реальную тяжесть поражения легких и служить прогностическим критерием перспективности ЭКМО.

По данным литературы, компьютерная томография (КТ) является одним из основных методов инструментальной диагностики паренхиматозных заболеваний легких, однако данных об использовании результатов КТ в прогностических целях немного. K. Ichikado et al. свидетельствуют, что наличие при КТ признаков фриброза легких в первую неделю после развития ОРДС является независимым предиктором летальности [35]. Сходные результаты получили J.H. Chung et al., по их данным, признаки фриброза при КТ, поражение более $80 \%$ объема легочной ткани и признаки повышения давления в правых отделах сердца (на основании КТ-признаков дилатации легочной артерии и увеличения объема правого желудочка) являются предикторами неблагоприятного исхода [36].

Время начала экстракорпоральной мембранной оксигенации

Практически во всех исследованиях и рекомендациях отмечается, что более раннее начало ЭКМО приводит к лучшим результатам. Причем оценивается не только время принятия решения от развития критической гипоксии до подключения ЭКМО, но и общее время от начала заболевания до развития тяжелого ОРДС. И если лучший 
результат в случае быстрого принятия решения вполне понятен, то причины худшего результата в случае медленного или отсроченного развития патологического процесса требуют дальнейшего изучения и осмысления.

Одним из ключевых критериев ОРДС, согласно Берлинской дефиниции, является время развития симптомов - не более 7 дней [37]. В то же время авторами шкалы RESP в качестве одного из предикторов благоприятного исхода предложено считать длительность ИВЛ до начала ЭКМО менее 48 часов. Таким образом, более медленное развитие заболевания само по себе является прогностически неблагоприятным критерием и может отложить принятие решения о начале ЭКМО.

Следует также отметить, что сроки развития ОРДС рассматриваются в качестве прогностических факторов и вне контекста ЭКМО. По данным Z. Ruyang et al. и R. Zhang et al. [38; 39], позднее начало ОРДС связано с более высокой 28- и 60-дневной летальностью. При этом медленное развитие симптомов наблюдалось у $31 \%$ пациентов, для них была характерна меньшая продолжительность жизни и более высокая летальность, чем у пациентов с более быстрым началом ОРДС. Кроме того, авторы также отмечают различия в динамике восстановления фрункции легких в указанных группах пациентов, подтверждая данные T.J. Iwashyna [40]. T.J. Iwashyna выделяют несколько «траекторий» поражения и восстановления (нарушения и восстановления фрункции) легких при ОРДС: «Big Hit - большой удар», «Slow Burn - медленное горение» и рекуррентное течение, практически неотличимое от предыдущего варианта. Траектория "Big Hit" характерна для пациентов с быстрым развитием ОРДС, проявляется быстрой утратой функции легких, которая в последующем постепенно восстанавливается. "Slow Burn" - функция легких, лишь умеренно сниженная вначале, прогрессивно снижается с развитием заболевания, приводя к худшему исходу - данный вариант характерен для медленного развития ОРдС.

Этиология острой дъхателъной недостаточности упоминается в руководствах в качестве возможного критерия оценки вероятности неблагоприятного исхода. Причины развития ОДН учитываются в шкале RESP [13], предложенной для прогнозирования исхода у пациентов с ОДН и являющейся, по мнению некоторых авторов, наиболее достоверной из предложенных [41].
Рассматриваются следующие заболевания, наиболее часто приводящие к ОДН: вирусная и бактериальная пневмонии, астма, травма и ожоги, аспирационный пневмонит и другие. Каждой нозологии или группе заболеваний соответствует определенное число баллов. Например, астматический статус считается более обратимым состоянием, чем бактериальная пневмония, что свидетельствует о лучшем прогнозе.

Сепсис нередко ассоциирован с развитием ОДН, в том числе и с ОРДС. При этом данные немногочисленных исследований не позволяют сделать однозначный вывод об эффективности ЭКМО при ОР ДС, вызванном сепсисом. N. Nessler et al. исследовали результаты применения ЭКМО у пациентов с интраабдоминальным гнойным процессом, при этом эфрфективность ЭКМО не была доказана [42]. S. Takauji et al. продемонстрировали эфрфективность ЭКМО при ОДН, вызванной пневмонией, однако у пациентов с ОдН, вызванной сепсисом внелегочной этиологии, не была продемонстрирована польза от ЭКМО [43].

Ни одна из причин респираторной недостаточности, даже те, что упомянуты в качестве необратимых (например, фриброз легких, эмсризема и др.) не являются абсолютным противопоказанием к проведению ЭКМО. Лечащему врачу предлагается самостоятельно принять решение о целесообразности ЭКМО, взвесив вероятность благоприятного исхода и возможности восстановления фрункции легких [14].

Обшая тяжесть состояния также может определять исход у больных с ОДН. Тяжелая сопутствующая патология в виде некурабельных состояний, например, тяжелого необратимого повреждения мозга или терминального онкологического процесса являются абсолютными противопоказаниями к проведению ЭКМО ввиду очевидного неблагоприятного прогноза. Использование ЭКМО при некоторых других заболеваниях может быть ограничено особенностями метода. Так, при проведении ЭКМО требуется гепаринизация ввиду повышенной вероятности тромбообразования, вызванной циркуляцией крови в экстракорпоральном контуре [11]. Поэтому наличие у пациента тяжелой сочетанной травмы или внутричерепного кровоизлияния может быть противопоказанием к ЭКМО.

При этом авторы некоторых рекомендаций склонны расширять перечень сопутствующих заболеваний, являющихся противопоказаниями к ЭКМО, например, в рекомендациях по про- 
ведению ЭКМО, опубликованных университетом Висконсина, к абсолютным противопоказаниям относятся полиорганная недостаточность с нарушением функции более чем трех систем огранов, диссекция аорты, тяжелая аортальная регургитация, а к относительным - печеночная недостаточность [17]. Критерии, отражающие общую тяжесть заболевания, используются и в указанных выше шкалах. Среди оцениваемых показателей в шкале PRESERVE фригурирует количество баллов по шкале SOFA. B шкале RESP учитывается дисфункция ЦНС и наличие перенесенной остановки кровообращения.

По данным A. Roch et al., количество баллов по шкале SOFA может быть использовано в качестве критерия для «точной оценки риска смерти у пациентов с ОРДС» [44].

Нарушения системы иммунитета фигурируют в качестве негативных прогностических критериев в шкалах RESP и PRESERVE. В рекомендациях ELSO относительным противопоказанием к ЭКМО считается иммуносупрессия с нейтропенией менее $400 /$ мм $^{3}$.
Заключение

Несмотря на увеличивающуюся частоту использования вено-венозной экстракорпоральной мембранной оксигенации для лечения острой дыхательной недостаточности, вопрос о прогнозировании исхода заболевания остается дискуссионным. Абсолютные и относительные противопоказания к проведению экстракорпоральной мембранной оксигенации, связанные с основным заболеванием и сопутствующей патологией, отличаются в разных рекомендациях. На настоящий момент предложено множество прогностических шкал и критериев, однако данные о применимости некоторых из них противоречивы.

В большинстве рекомендаций анализируются косвенные критерии тяжести, такие как возраст пациента, сроки болезни, сопутствующие заболевания, однако детального анализа состояния легких не проводится.

Отсутствие общепринятых критериев обратимости патологического процесса в легких существенно затрудняет оценку соотношения пользариск при принятии решения о начале проведения экстракорпоральной мембранной оксигенации.

\section{Литература / References}

1. Evseev AK, Zhuravel SV, Alentiev AYu, Goroncharovskaya IV, Petrikov SS. Membranes in extracorporeal blood oxygenation technology. Membranes and Membrane Technologies. 2019;1(4):201211. https://doi.org/10.1134/ S2517751619040024

2. Hill JD, O'Brien TG, Murray JJ, Dontigny L, Bramson ML, Osborn JJ, et al. Prolonged extracorporeal oxygenation for acute post-traumatic respiratory failure (shock-lung syndrome). Use of the Bramson membrane lung. $N$ Engl $J$ Med. 1972;286(12):629-634. PMID: 5060491 https://doi.org/10.1056/ NEJM197203232861204

3. Zapol WM, Snider MT, Hill JD, Fallat RJ, Bartlett RH, Edmunds LH, et al.
Extracorporeal membrane oxygenation in severe acute respiratory failure: a randomized prospective study. JAMA. 1979;242(20):2193-2196. PMID: 490805 https://doi.org/10.1001/jama.242.20.2193 4. Davies A, Jones D, Bailey M, Beca J, Bellomo R, Blackwell N, et al. Extracorporeal membrane oxygenation for 2009 influenza A (H1N1) acute respiratory distress syndrome. JAMA. 2009;302(17):1888-1895. PMID: 19822628 https://doi.org/10.1001/jama.2009.1535 5. Noah MA, Peek GJ, Finney SJ, Griffiths MJ, Harrison DA, Grieve R, et al. Referral to an extracorporeal membrane oxygenation center and mortality among patients with severe 2009 influenza A(H1N1). JAMA. 2011;306(15):1659-
1668. PMID: 21976615 https://doi. org/10.1001/jama.2011.1471

6. Журавель С.В., Косолапов Д.А., Кецкало М.В. Организация программы экстракорпоральной мембранной оксигенации у взрослых пациентов в многопрофильном стационаре. Опыт Регенсбурга (Германия). Трансплантология. 2014;(4):28-32. Zhuravel SV, Kosolapov DA, Ketskalo MV. The organization of extracorporeal membrane oxygenation programs for acute respiratory failure in adult patients in a multidisciplinary hospital. Review of experience in Regensburg (Germany). Transplantologiya. The Russian Journal of Transplantation. 2014;(4):28-32. (In Russ.).

7. Combes A, Hajage D, Capellier G, 
Demoule A, Lavoué S, Guervilly C, et al. Extracorporeal membrane oxygenation for severe acute respiratory distress syndrome. N Engl J Med. 2018;378(21):19651975. PMID: 29791822 https://doi. org/10.1056/NEJMoa1800385

8. Курилова О.А., Журавель С.В., Романов А.А., Маринин П.Н., Цурова Д.Х., Каллагов Т.Э. и др. Опыт применения экстракорпоральной мембранной оксигенации для обеспечения двусторонней трансплантации легких. Вестник трансплантологии и искусственных органов. 2014;(2):66-74. Kurilova OA, Zhuravel SV, Romanov AA, Marinin PN, Tsurova DKh, Kallagov TE, et al. Experience with application of extracorporeal membrane oxygenation in double lung transplantation. Russian Journal of Transplantology and Artificial Organs. 2014;(2):66-74. (In Russ.). https://doi. org/10.15825/1995-1191-2014-2-66-74 9. Peek GJ, Mugford M, Tiruvoipati R, Wilson A, Allen E, Thalanany MM, et al. Efficacy and economic assessment of conventional ventilatory support versus extracorporeal membrane oxygenation for severe adult respiratory failure (CESAR): a multicentre randomised controlled trial. Lancet. 2009;374:13511363. PMID: 19762075 https://doi. org/10.1016/S0140-6736(09)61069-2

10. Munshi L, Walkey A, Goligher E, Pham T, Uleryk EM, Fan E. Venovenous extracorporeal membrane oxygenation for acute respiratory distress syndrome: a systematic review and meta-analysis. Lancet Respir Med. 2019;7(2):163172. PMID: 30642776 https://doi. org/10.1016/S2213-2600(18)30452-1

11. Бахарев С.А., Попугаев К.А., Киселев К.В., Самойлов А.С., Удалов Ю.Д., Жангазинов А.Л. и др. Механизмы развития геморрагических осложнений при проведении экстракорпоральной мембранной оксигенации. Пилотное исследование. Анестезиология и реаниматология. 2020;(1):25-34. Bakharev SA, Popugaev KA, Kiselev KV, Samoylov AS, Udalov $\mathrm{YuD}$, Zhangazinov AL, et al. Mechanisms of hemorrhagic complications during extracorporeal membrane oxygenation. A pilot study. Russian Journal of Anaesthesiology and Reanimatology = Anesteziologiya $i$ reanimatologiya 2020;(1):25-34. (In Russ.). https://doi. org/10.17116/anaesthesiology202001125 12. Schmidt M, Zogheib E, Rozé $H$, Repesse X, Lebreton G, Luyt CE. The PRESERVE mortality risk score and analysis of long-term outcomes after extracorporeal membrane oxygenation for severe acute respiratory distress syndrome. Intensive Care Med. 2013;39(10):1704-1713. PMID: 23907497 https: //doi.org/10.1007/s00134-0133037-2

13. Schmidt M, Bailey M, Sheldrake J, Hodgson C, Aubron C, Rycus PT, et al. Predicting survival after extracorporeal membrane oxygenation for severe acute respiratory failure. The Respiratory extracorporeal membrane oxygenation survival prediction (RESP) Score. Am J Respir Crit Care Med. 2014;189(11):13741382. PMID: 24693864 https://doi. org/10.1164/rccm.201311-2023OC

14. ELSO Guidelines for cardiopulmonary extracorporeal life support extracorporeal life support organization, Version 1.4 August 2017 Ann Arbor, MI, USA. Available at: https://www.elso. org/Portals / / ELSO $\% 20$ Guidelines $\% 20$ General\%20All\%20ECLS\%20Version\%20 1_4.pdf [Accessed March 23, 2019]

15. Sladen RN. ECMO in the adult patient, core critical care. Anesthesia Analgesia. 2018;126(2):713. https://doi. org/10.1213/ANE.0000000000002675

16. Sangalli F, Patroniti N, Pesenti A. (eds.) ECMO-Extracorporeal life support in adults. Switzerland AG: Springer; 2014.

17. Extracorporeal Membrane Oxygenation (ECMO): Initiation and management-adult - in patient: clinical practice guideline. USA: University of Wisconsin Hospitals and Clinics Authority; 2018.

18. Papazian L, Aubron C, Brochard L, Chiche JD, Combes A, Dreyfuss D, et al. Formal guidelines: management of acute respiratory distress syndrome. Ann Intensive Care. 2019;9(1):69. PMID: 31197492 https://doi.org/10.1186/ s13613-019-0540-9

19. Villar J, Blanco J, del Campo $R$, Andaluz-Ojeda D, Díaz-Domínguez FJ, Muriel A, et al. Assessment of $\mathrm{PaO}_{2} / \mathrm{FiO}_{2}$ for stratification of patients with moderate and severe acute respiratory distress syndrome. BMJ Open. 2015;5(3):e006812. PIMID: 25818272 https://doi.org/10.1136/ bmjopen-2014-006812

20. Villar J, Ambrós A, Soler JA, Martínez D, Ferrando C, Solano R, et al. Age, $\mathrm{PaO}_{2} / \mathrm{FiO}_{2}$, and plateau pressure score: a proposal for a simple outcome score in patients with the acute respiratory distress syndrome. Crit Care Med. 2016;44(7):1361-1369. PMID: 27035239 https://doi.org/10.1097/ CCM.0000000000001653
21. Heiss KF, Bartlett RH. Extracorporeal membrane oxygenation: an experimental protocol becomes a clinical service. Adv Pediatr. 1989;36:117-136. PMID: 2675567 22. Dechert RE, Park PK, Bartlett RH. Evaluation of the oxygenation index in adult respiratory failure. $J$ Trauma Acute Care Surg. 2014;76(2):469-473. PMID: 24458052 https://doi.org/10.1097/ TA.0b013e3182ab0d27

23. Chen WL, Lin WT, Kung SC, Lai CC, Chao CM. The value of oxygenation saturation index in predicting the outcomes of patients with acute respiratory distress syndrome. J Clin Med. 2018;7(8). pii: E205. PMID: 30096809 https://doi. org $/ 10.3390 / \mathrm{jcm} 7080205$

24. Murray JF, Matthay MA, Luce JM, Flick MR. An expanded definition of the adult respiratory distress syndrome. Am Rev Respir Dis. 1988;138(3):720-723. PMID: 3202424 https://doi.org/10.1164/ ajrccm/138.3.720

25. Kangelaris KN, Calfee CS, May AK, Zhuo H, Matthay MA, Ware LB. Is there still a role for the lung injury score in the era of the Berlin definition ARDS? Ann Intensive Care. 2014;4(1):4. PMID: 24533450 https://doi.org/10.1186/21105820-4-4

26. DesPrez K, McNeil JB, Wang C, Bastarache JA, Shaver CM, Ware LB, Oxygenation saturation index predicts clinical outcomes in ARDS. Chest. 2017;152(6):1151-1158. PMID: 28823812 https://doi.org/ 10.1016/j. chest.2017.08.002

27. Schmidt M, Pham T, Arcadipane A, Agerstrand C, Ohshimo S, Pellegrino V, et al. Mechanical ventilation management during extracorporeal membrane oxygenation for acute respiratory distress syndrome: an international multicenter prospective cohort. Am J Respir Crit Care Med. 2019;200(8):1002-1012. PMID: 31144997 https://doi.org/10.1164/ rccm.201806-1094OC

28. Dreyfuss D, Soler P, Basset G, Saumon G. High inflation pressure pulmonary edema. Respective effects of high airway pressure, high tidal volume, and positive end-expiratory pressure. Am Rev Respir Dis. 1988;137(5):1159-1164. PMID: 3057957 https://doi.org/10.1164/ ajrccm/137.5.1159

29. Gattinoni L, Pesenti A. The concept of "baby lung". Intensive Care Med. 2005;31(6):776-784. PMID: 15812622 https://doi.org/10.1007/s00134-005$2627-\mathrm{z}$

30. Fan E, Del Sorbo L, Goligher EC, 
Hodgson CL, Munshi L, Walkey AJ, et al. An Official American thoracic society/ European society of intensive care medicine/Society of critical care medicine. Clinical practice guideline: Mechanical ventilation in adult patients with acute respiratory distress syndrome. Am J Respir Crit Care Med. 2017;195(9):12531263. PMID: 28459336 https://doi. org/10.1164/rcem.201703-0548ST

31. Amato MB, Meade MO, Slutsky AS, Brochard L, Costa EL, Schoenfeld DA, et al. Driving pressure and survival in the acute respiratory distress syndrome. $N$ Engl J Med. 2015;372(8):747-755. PMID: 25693014 https://doi.org/10.1056/ NEJMsa1410639

32. Kim HS, Kim JH, Chung CR, Hong SB, Cho WH, Cho YJ, et al. Lung compliance and outcomes in patients with acute respiratory distress syndrome receiving ECMO. Ann Thorac Surg. 2019;108(1):176-183. PMID: 30836100 https://doi.org/10.1016/j.athoracsur.2019.01.055

33. Rozé H, Doassans G, Repusseau B, Ouattara A. Decrease of thoracopulmonary compliance with pressure assist controlled ventilation in ARDS patients under ECMO and transported to a referral centre. Intensive Care Med. 2017;43(1):148-149. PMID: 27815586 https://doi.org/10.1007/s00134-0164616-9

34. Walkey AJ, Goligher EC, Del Sorbo L, Hodgson CL, Adhikari NKJ, Wunsch H, et al. Low tidal volume versus non-volume-limited strategies for patients with acute respiratory distress syndrome: a systematic review and meta-analysis. Ann Am Thorac Soc. 2017;14(Suppl 4):S271-S279. PMID: 28846440 https://
doi.org/10.1513/AnnalsATS.201704$337 \mathrm{OT}$

35. Ichikado K, Suga M, Muranaka H, Gushima Y, Miyakawa H, Tsubamoto M, et al. Prediction of prognosis for acute respiratory distress syndrome with thin-section $\mathrm{CT}$ : validation in 44 cases. Radiology. 2006;238(1):321-329. PMID: 16293804 https://doi.org/10.1148/radiol.2373041515

36. Chung JH, Kradin RL, Greene RE, Shepard JA, Digumarthy SR. CT predictors of mortality in pathology confirmed ARDS. Eur Radiol. 2011;21(4):730737. PMID: 20927526 https://doi. org/10.1007/s00330-010-1979-0

37. Ranieri VM, Rubenfeld GD, Thompson BT, Ferguson ND, Caldwell E, Fan E, et al. The ARDS definition task force. Acute respiratory distress syndrome: the Berlin definition. JAMA. 2012;307(23):2526-2533. PMID: 22797452 https://doi.org/10.1001/jama.2012.5669 38. Ruyang $\mathrm{Z}$, Zhaoxi W, Yongyue $\mathrm{W}$, Paula TA, Zhaozhong Z, Li S, et al. A two-stage association study identifies a high risk window of acute respiratory distress syndrome onset time correlated with poor 28 day overall survival. A53. Respiratory failure: risk factors and outcomes in ARDS. American Thoracic Society - 2016, (California, San-Francisco, 13-18 may 2016). San-Francisco, 2016. Abstr A7794.

39. Zhang R, Wang Z, Tejera P, Frank AJ, Wei Y, Su L, et al. Late-onset moderate to severe acute respiratory distress syndrome is associated with shorter survival and higher mortality: a two-stage association study. Intensive Care Med. 2017;43(3):399-407. PMID: 28032130 https://doi.org/10.1007/s00134-016-
4638-3

40. Iwashyna TJ. Trajectories of recovery and dysfunction after acute illness, with implications for clinical trial design. Am $J$ Respir Crit Care Med. 2012;186(4):302304. PMID: 22896591 https://doi. org/10.1164/rccm.201206-1138ED

41. Rozencwajg S, Pilcher D, Combes A, Schmidt M. Outcomes and survival prediction models for severe adult acute respiratory distress syndrome treated with extracorporeal membrane oxygenation. Crit Care. 2016;20(1):392 PMID: 27919283 https://doi.org/10.1186/ s13054-016-1568-y

42. Nesseler $\mathrm{N}$, Launey $\mathrm{Y}$, Isslame $\mathrm{S}$, Flécher E, Lebouvier T, Mallédant Y, et al. Is extracorporeal membrane oxygenation for severe acute respiratory distress syndrome related to intra-abdominal sepsis beneficial? Intensive Care Med. 2015;41(5):943-945. PMID: 25800583 https://doi.org/10.1007/s00134-0153743-z

43. Takauji S, Hayakawa M, Ono K, Makise H. Respiratory extracorporeal membrane oxygenation for severe sepsis and septic shock in adults: a propensity score analysis in a multicenter retrospective observational study. Acute Med Surg. 2017;4(4):408-417. PMID: 29123901 https://doi.org/10.1002/ams2.296

44. Roch A, Hraiech S, Masson E, Grisoli D, Forel JM, Boucekine M, et al. Outcome of acute respiratory distress syndrome patients treated with extracorporeal membrane oxygenation and brought to a referral center. Intensive. Intensive Care Med. 2014;40(1):74-83. PMID: 24170143 https://doi.org/10.1007/s00134-0133135-1 


\section{BEVIEW ARTICLES AND LEQTURES}

\section{Информация об авторах}

Павел Александрович Брыгин

\section{Сергей Владимирович Журавель}

\section{Даниил Александрович Троицкий}

Ирина Игоревна
Уткина

канд. мед. наук, заведующий отделением реанимации и интенсивной терапии для ожоговых больных ГБУЗ «НИИ скорой помощи им. Н.В. Склифросовского ДЗМ», https://orcid.org/0000-0002-8511-9646 $30 \%$ - написание текста рукописи

д-р мед. наук, заведующий научным отделением анестезиологии, реаниматологии и интенсивной терапии ГБУЗ «НИИ скорой помощи им. Н.В. Склифосовского ДЗМ», https://orcid.org/0000-0002-9992-9260 $30 \%$ - обзор публикаций по теме статьи

врач-ординатор отделения анестезиологии, реаниматологии и интенсивной терапии ГБУЗ «НИИ скорой помощи им. Н.В. Склифосовского ДЗМ», https:// orcid.org/0000-0002-1181-518X

$20 \%$ - обзор публикаций по теме статьи

канд. мед. наук, старший научный сотрудник отделения анестезиологии, реаниматологии и интенсивной терапии ГБУЗ «НИИ скорой помощи им. Н.В. Склифосовского ДЗМ», https://orcid.org/0000-0002-5685-4916

$20 \%$ - написание текста рукописи

\section{Information about authors}

Pavel A. Brygin

Cand. Med. Sci., Head of the Intensive and Critical Care Unit for Burn Patients, N.V. Sklifosovsky Research Institute for Emergency Medicine, https://orcid. org/0000-0002-8511-9646

$30 \%$ - writing the text of the manuscript

Dr. Med. Sci., Head of the Scientific Department for Anesthesiology, Intensive and

Sergey V. Zhuravel Critical Care, N.V. Sklifosovsky Research Institute for Emergency Medicine, https:// orcid.org/0000-0002-9992-9260 $30 \%$ - review of publications on the topic of the article

Resident Physician of the Department for Anesthesiology, Intensive and Critical Care, N.V. Sklifosovsky Research Institute for Emergency Medicine, https://orcid. org/0000-0002-1181-518X $20 \%$ - review of publications on the topic of the article

Cand. Med. Sci., Senior Researcher, Department for Anesthesiology, Intensive and

Irina I. Utkina Critical Care, N.V. Sklifosovsky Research Institute for Emergency Medicine, https:// orcid.org/0000-0002-5685-4916

$20 \%$ - writing the text of the manuscript

Статья поступила: 15.04.2020

Received: April 15, 2020

Статья принята в печать: 27.04.2020

Accepted for publication: April 27, 2020 